\title{
Dynamic Virtual Network Embedding based on Auction-Game Theory
}

\section{NingNing Liang ${ }^{1}$, JuLong Lan ${ }^{1}$, YunFu Long ${ }^{2}$, GuanYing Liu ${ }^{2}$}

\author{
${ }^{1}$ National Digital Switching System Engineering \& Technological Research \& Development Center, \\ Zhengzhou, 450002, China
}

${ }^{2}$ The Air Defence Forces Academy, Zhengzhou, 450052, China

Keywords: virtual network, auction-game, business request.

\begin{abstract}
To solve the problem of competition among different business sharing the limited substrate network resource, this paper provides a construction model based on auction-game theory. The model regards business as players and business requests as strategy space. Based on this model, a dynamic algorithm of virtual network embedding using auction-game theory is proposed, which can maximize the whole construction effectiveness of the virtual network. The experimental results show that the proposed algorithm has better performance than the existing algorithms, in terms of the whole construction effectiveness, the construction success rate and the occupancy rate of substrate resource.
\end{abstract}

\section{Introduction}

Network virtualization is recognized as a promising solution to the network ossification problem. It allows multiple virtual networks (VN) to coexist the shared physical infrastructure according to service requests and the statement of substrate network resource ${ }^{[1]}$. As shown in Fig.1.

Virtual network embedding (VNE) is a process that maps the business request on the corresponding physical path and allocates bandwidth according to some constraint. And its essence is the problem of substrate resource allocation. The existing methods have two problems. Firstly, current construction methods generally assume that different business requests are separated mutually when asking for the limited resources concurrently, but neglect the competitions among them. Secondly, the existing algorithms implement remapping while substrate nodes or links failure, or new arrival request being refused, but have not consider dynamic construction based on different business scenarios.

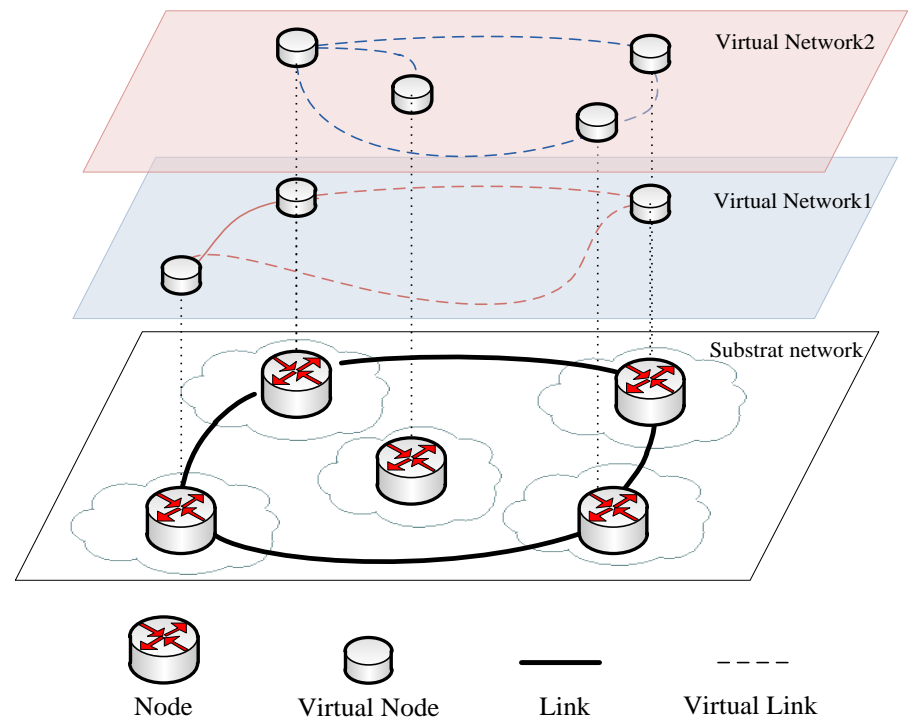

Fig.1 Sketch map of VNE

For solving the above problems, this paper provides a dynamic algorithm of VNE based on auction-game theory (DAG-VNE). The procedure is shown in Fig.2. Firstly, each business request asks for its construction requirement, then calculates the payment of every business request based on the auction mechanism and returns it to the business request, finally we introduce the 
cooperative game theory which maximum the effectiveness of system to allocate the substrate resources.

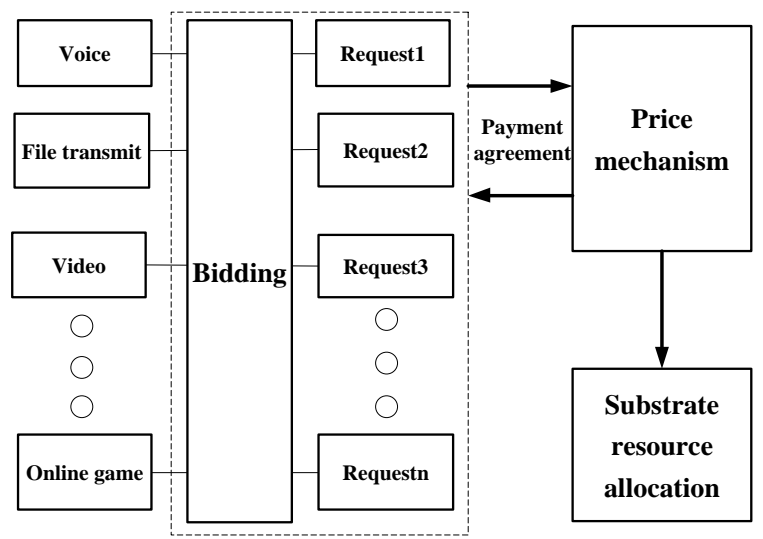

Fig.2 VNE based on auction-game theory

This paper is organized as follows. In the next section, the existing VN embedding algorithms are reviewed. In Section III, our DAG-VNE algorithm is introduced in detail. In Section IV, DAG-VNE is compared with other algorithms via simulations. Finally, we conclude the paper in Section V.

\section{Related work}

Current studies on VNE are concentrated on resource allocation and network mapping ${ }^{[2,3]}$. Zhu ${ }^{[4]}$ presents an algorithm which is aiming at load balance problem to map the virtual node onto the relative nearer and lighter load substrate node, and then map the virtual link by means of the shortest path. $\mathrm{He}^{[5]}$ provides a distributed mapping algorithm which coordinates the virtual node and virtual link mapping, but there is a wide gap between centralized algorithms. And it doesn't take the life time of dynamic VN requests in consideration. Chowdhury ${ }^{[6]}$ provides a mathematical programming of coordinating node and link mapping, reducing the searching space by providing virtual node location. To improve the acceptance rate of VN and maximize the cost-effectiveness, researchers are concerning the game theory increasingly. But most of them ${ }^{[7-9]}$ just consider video request instead of building a general model.

\section{Dynamic virtual network embedding based on auction-game theory}

\section{Virtual network model}

Substrate resource. We abstract the substrate network into undirected graph $G^{s}=\left(N^{s}, L^{s}, C^{s}\right)$, where $N^{s}$ and $L^{s}$ represent a set of substrate nodes and links. $C^{s}$ Denotes substrate resources, includes node resources and link resources. Meanwhile, we introduce link resource unit cost $C_{\text {link }}$ and node resource unit cost $C_{\text {node. }}$.

Virtual network request model. The virtual network request (VNR) is expressed as an undirected graph $G^{r}=\left(N^{r}, L^{r}, R^{r}\right)$, where $N^{r}$ and $L^{r}$ represent the set of virtual nodes and virtual links, and they are subset of $N^{\mathrm{S}}$ land $L^{\mathrm{S}}$. $R^{r}$ Denotes the constraint to virtual nodes and virtual links. In the meantime, VNR also includes request functions, contains the quantity of resource willing to buy, the price and the service time, and so on.

Virtual network embedding. VNE can be expressed as a mapping which satisfies the constraint $R^{r}$ and a subset from $G^{r}$ to $G^{s}$ in undirected graph $G^{r}$.

$$
M: G^{r} \mapsto G^{v}, G^{v}=\left(N^{v}, L^{v}, C^{v}\right)
$$

Where $N^{v}$ and $L^{v}$ are the subset of $N^{\mathrm{s}}$ and $L^{\mathrm{s}}$, respectively. $G^{v}$ Represents the service ability of substrate network. The VNE can be divided into node mapping and ling mapping. Node Mapping: $M^{N}:\left(N^{r}, R_{N}^{r}\right) \mapsto\left(N^{v}, C_{N}^{v}\right)$

Link Mapping $M^{L}:\left(L^{r}, R_{L}^{r}\right) \mapsto\left(L^{v}, C_{L}^{v}\right)$; 
Where $R_{N}^{r}$ and $R_{L}^{r}$ represent the constraints to the virtual nodes and virtual links. $C_{N}^{v}$ And $C_{L}^{v}$ denote service supporting ability of substrate physical nodes and links, respectively.

Objective. For the limited substrate resources, the purpose of VNE is to maximize utilization of substrate resources and the total effectiveness.

\section{Construction of game theory model}

Element of the model. Players: Business is defined as game players, and denoted as $N=\{1,2$, 3...n\}.

Strategy: We construct the set of strategy space $C=\left\{B w, D_{l}, C_{b m}, C P_{c p u}\right\}$, where $B w$ represents bandwidth, and $D_{l}$ reprints delay, those are the corresponding VN link request. Node requests are denoted by $C_{b m}$ and $C P_{c p u}$, respectively, where $C_{b m}$ represents buffer memory capacity, and $C P_{c p u}$ represents CPU calculation capability.

Utility function: The utility function is defined as the maximized embedding total revenue.

$$
R\left(G^{S}\right)=\sum_{n \in G^{\varphi}} P_{n}-\sum_{G^{S}} C\left(C_{\text {link }}, C_{\text {node }}\right)=\sum_{n \in G^{\nu}} P_{n}-\sum_{G^{S}}\left(C_{\text {link }} f_{1}\left(\frac{B w}{D_{l}}\right)+C_{\text {node }} f_{2}\left(C_{b m}, C P_{c p u}\right)\right)
$$

Where $\sum_{n \in G^{y}} P_{n}$ represents the sum of payment, and $\sum_{G^{S}} C\left(C_{\text {link }}, C_{\text {node }}\right)$ represents the cost of mapping.

Thus, the maximized total revenue can be expressed as follows.

$$
\begin{aligned}
& \max R\left(G^{S}\right) \\
& \text { s.t. } P_{n}>C\left(C_{\text {link }}, C_{\text {node }}\right), \quad d_{p}<D_{l}, \quad \sum_{n=1}^{N} C_{b m_{n}}^{r}<C_{b m}^{s}, \quad \sum_{n=1}^{N} C P_{c u_{n}}^{r}<C P_{c p u}^{s}, \quad \sum_{n=1}^{N} B w_{n}^{r}<Q
\end{aligned}
$$

Where, Constraint (4) represents that the bidding of VNR must be more than substrate resources cost, the delay of selected path must less than delay requirement of VNR, and the sum of resource which is consumed by virtual nodes or links must less than the maximal capacity of substrate nodes and links.

Pricing mechanism

In this paper, we design our algorithm based on auction pricing mechanism, and assume all of the business requests truly express their own requirements of VNE.

Business request function: The business request function $q_{n}\left(P_{n}, t_{n}\right)$ is the $n$th business willing to pay $P_{n}$ for its resource requirements $q_{n}$ during the used time $t_{n}$, and denotes as $q_{n}\left(P_{n}, t_{n}\right)=\left\{t_{n}, P_{n}, q_{n}\right\}$.

Auction rules: We suppose that $n$ businesses ask for requests of VNE, competing for the total substrate resources of $Q$ in a certain time period T. Given the different business requests payment $P_{n}$, and $q_{n}$ which the quantity of $n$th business willing to buy, we determine the resource unit price in a certain time period $\mathrm{T}$ according to the bidding offered by business.

$$
p(\mathrm{~T})=\max \left\{\frac{q_{n}}{P_{n}} \mid \sum_{n=1}^{N} q_{n}\left(P_{n}, t_{n}\right) \leq Q\right\}
$$

Then we can get that, $n$th business obtains resource:

Nth business needs to pay for:

$$
q_{n}(T)=q_{n}(p(T))
$$

$$
c_{n}=P(T) \cdot q_{n}(P(T))
$$

We get the market clearing price from formula (5) when the bidding $q_{n}\left(P_{n}, t_{n}\right)$ is real, it will enable the optimum resource allocation. When all players know the number of business $n$ and the total quantity of resource $Q$, and moreover, $Q$ is invariant, there exists Nash equilibrium in the auction ${ }^{[10 \text {, }}$ 11].

\section{Dynamic virtual network embedding based on auction-game theory}

We assume there are $n$ business requests in time period T. We determine the fee that a certain business needs to pay, and feed it back to the corresponding business by auction mechanism. And we will map VN for the business which accepts the charge. In VNE, firstly we build VNRs into set $Q_{n}$ and cluster it into subset $Q_{i}$ according to the business types, aware the resource information of the substrate network, figure out all paths which satisfy the VNRs, then put each path into formula(2), get the optimum path and bandwidth. So far, we map subset $Q_{i}$ to the substrate network which satisfy VNRs, and accomplish VNE. The flow chart of the algorithm is as Fig.3 shown. 


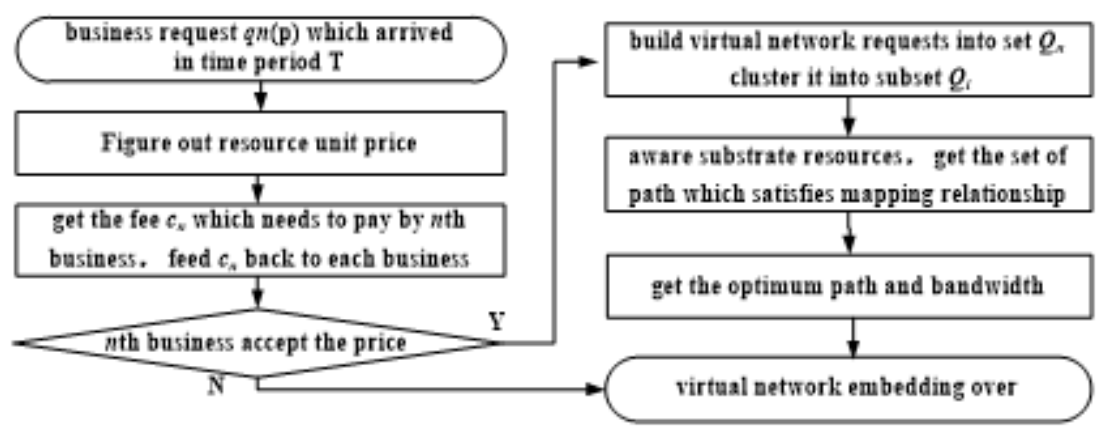

Fig.3 Flow chart of DAG-VNE

\section{Simulation and performance analysis}

For evaluating performance of the proposed algorithm, we compare it with the algorithm ViNE ${ }^{[6]}$ which is based on the simple-economic model and coordinates virtual nodes and links mapping, as well as the algorithm G-SP ${ }^{[4]}$ which targets to load balance.

\section{Experimental environment}

Substrate network topology of the experiment consists of 150 nodes which are produced randomly by BRITE. Nodes connection rate is 0.5 . Node and link resources are distributed uniformly between 50 and 100. The number of VNR nodes is distributed uniformly between 2 and 10. Link bandwidth, node CPU capacity and memory capacity are all in uniform distribution between 0 and 30. Nodes connection rate is 0.5. VNRs arrive in a Poisson process with an inter-arrival time of 100 time units and intensity is 10. Lifetimes of the VNRs follow an exponential distribution with mean 1000 time units. The mapping period is unit time $\mathrm{T}$. The unit resource bidding of business requests is distributed uniformly between 1 and 3, and the unit cost of under layer nodes and links is 1 . We assume the business will accept the price as long as the resource unit price which is figured out by the auction mechanism. In order to ensure the accuracy of simulation result, all the results presented for an experiment are an average of 20 runs of simulation.

Simulation results and analysis

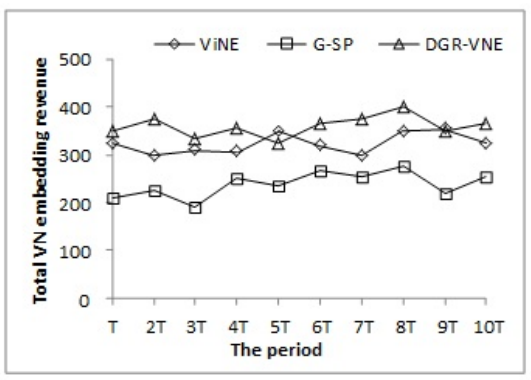

Fig.4 Total embedding revenue

Fig.4 shows the total VNE revenue of different algorithms. As shown in Fig.4, DAG-VNE algorithm performs better than other algorithms. Even though G-SP aims at load balance, but it divides the mapping into two phases, so that greatly limits the scheme space, and its total revenue is slightly lower than ViNE which considers the node mapping and the link mapping simultaneously. However, because of igniting the competition among business by the auction mechanism, DAGVNE brings higher revenue. 


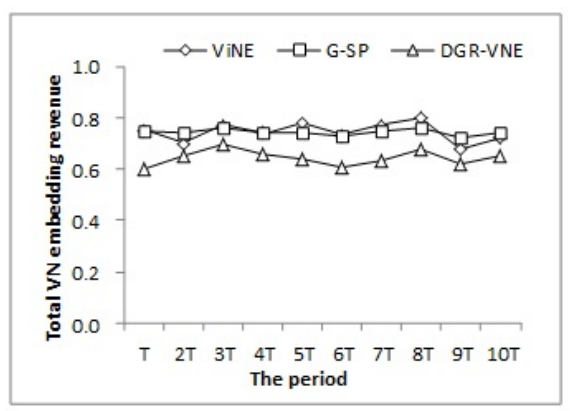

Fig. 5 embedding success rate

Fig. 5 shows the embedding success rate in different time periods. ViNE coordinates virtual node mapping and virtual link mapping into one phase, so its success rate is higher than the G-SP which is on the basis of separating the node mapping and link mapping into two phases. In order to maximum the total revenue, DAG-VNE has to two-way selection while pricing. As we set in the experiment that the business doesn't accept the price exceeding the double of its bidding, some business may voluntarily give up their requests at the beginning of the mapping. So the success rate is approximately $75 \%$, relatively lower than ViNE.

\section{Conclusions}

In this paper, we present a dynamic algorithm of VNE based on the auction-game theory. On the basis of the competition of various applications sharing the limited substrate network resource, the proposed algorithm maps $\mathrm{VN}$ according to the business request and aware the change of the business timely, dynamically adjusts the mapped $\mathrm{VN}$, and maximizes the profits of the whole system ultimately.

\section{Acknowledgements}

The research work was supported by National Basic Research Program of China No. 2012CB315901 and No. 2013CB329104, National Nature Science Foundation of China No. 61372121, National High Technology Research and Development Program of China No. 2013AA013505.

\section{References}

[1] Andreas Fischer et al., Virtual Network Embedding: A Survey, IEEE Communications Surveys \& Tutorials, 2013: p.1888-1906.

[2] Rafael L. Gomes et al., A Bandwidth-Feasibility Algorithm for Reliable Virtual Network Allocation, IEEE 28th International Conference on Advanced Information Networking and Applications, 2014: p.504- 511.

[3] Sarah Kareche et al., A New Robust Heuristic for Assigning Substrate Network Resources to Virtual Networks, International Conference on Advanced Networking Distributed Systems and Applications, 2014: p. 47- 52.

[4] Y. Zhu and M. Ammar, Algorithms for assigning substrate network resources to virtual network components, In Proc. IEEE INFOCOM, 2006: 1- 12.

[5] J. He et al., Davinci: dynamically adaptive virtual networks for a customized internet, CoNEXT, 2008: p. 9-12.

[6] N. Chowdhury, M. Rahman and R. Boutaba, ViNEYard: Virtual Network Embedding Algorithms with Coordinated Node and Link Mapping, ACM/IEEE Transaction on Networking, 2011: p. 206- 219.

[7] Jian Guo et al., A Cooperative Game Based Allocation for Sharing Data Center Networks, 2013. 
[8] Jun He et al., A Fast, Simple and Near-optimal Content Placement Scheme for a Large-scale VoD System, 2012.

[9] Yuan Feng and Bo Li, Bargaining Towards Maximized Resource Utilization in Video Streaming Datacenters, 2012.

[10] R .Wilson, Auctions of shares Quarterly Journal of Economics, 1979: p. 675- 698.

[11] K .Back and J. F. Zender, Auctions of divisible goods: on the rationale for the Treasury experiment, Review of Financial Studies, 1993: p. 733- 764. 